very great difficulty in securing, which was only accomplished by making a still freer incision and picking up both ends and tying. The wound was then washed with a strong solution of chloride of zinc, dusted with iodoform, a long drainage-tube inserted, closed with sutures, and dressed with antiseptic gauze. The portion of omentum removed, when spread out, was as large as the palm of the hand.

Jan. 30th.-The patient passed a fairly good night, although troubled by cough at times, which was treated with an occasional dose of linctus morphix. She had some little pain in the wound, and took three half-orain opium pills during the night and a little milk and limewater.-9 A.M.: Pulse 104; temperature 101.4\%. Some slight abdominal tenderness.-2 P.M.: Wound dressed looking well; a little blood-coloured inodorous discharge.

31st.-9 A.M.: Pulse 96; temperatnre $1002^{\circ}$; the temperature during the night had reached $102 \cdot 2^{\circ}$; has a little bronchial catarrh. Ordered an ounce of compound draught of senega every four hoúrs. Wound again dressed; looking well, but had some slight tenderness over abdomen.

Feb. 2nd.-9 A.M.: Pulse 100; temperature 100.8

3rd.-Pulse 100; temperature $100^{\circ}$. From this date the patient improved, the wound granulating well. The drainagetubes were removed, and on March 17 th she was walking about the ward wearing a truss and in good health.

STRANGULATED UMBILICAL HERNIA; OPERATION; REMOVAL OF THE SAC AFTER LIGATURE OF THE NECK; RECOVERY.

(Under the care of Mr. J. W. HuLkE.)

E. 0 - a man aged sixty-one years, was admitted at 9 P.M. on March 3rd, 1885, complaining of some vomiting and constipation, and a large swelling about the umbilicus. He stated that for five years he had suffered from hernia, but that he could always reduce it easily; for the last two years he had worn a belt, and there had always been more or less swelling. On March 1st, without any apparent cause, he experienced pain in the abdomen, and noticed that the swelling was larger; he vomited after taking any food. on the 2nd he placed himself under medical treatment. Ice was tried, and an attempt at reduction made, but this failed, and, the vomiting and constipation continuing, he was advised to come to the hospital; but twelve hours previous to admission he took a large dose of castor oil and fomented the rupture on his own responsibility. This failing to act, he sought admission.

On admission there was a large swelling about the size of the clenched fist in the umbilical region. Its upper surface was almost entirely denuded of epithelium from the application of poultices, ice, \&c. On palpation the swelling was found to extend some distance beneath the skin, more than was apparent to the eye. It was very hard, gave no impulse on coughing, was irreducible, but only very slightly tender on manipulation. It was diagnosed to be strangulated umbilical hernia.

Mr. Hulke, being in the hospital, decided to place the patient under ether at once, and if taxis failed-which it did - to operate at once. The parts were first washed out with carbolic solution, and iodoform dusted into the umbilicus. A semicircular incision was made at the upper and left outer margin of the swelling, with the intention of dissecting down to the constriction, and reducing, if possible, without opening the sac; but after carrying the dissection down a considerable distance, it was found impossible to do this, and another incision was made, running outwards and upwards from the centre of the umbilicus, and cutting the previous one at right angles. The strictures were then rery carefully divided until a deep red and congested tissue, which looked and felt exactly like the bowel, came into riew, but the finger could not be passed round the swelling; and the incision was further increased by two diverging ones, and then beneath this thickened membrane a bubble of air and fluid could be seen, showing evidently that this was the sac, and it was consequently picked up and pricked, and a large quantity of blood-coloured watery fluid escaped, showing the sac had been opened. It was freely dirided, and a large piece of omentum deeply congested came into view; this was pulled forward, and numerous double ligatures passed through and firmly tied; the mass was then cut away (it weighed twelve ounces). The finger was next passed down to the constriction, which was found to be very tight and fibrous, and was divided with a hernia knife; a few bleeding points were ligatured. The whole surface was then thoroughly washed with car- bolic solution ( 1 in 20 ), and there being no bleeding whatever, the stump was pocketed. The sac was next dealt with, and was found to be adherent nearly throughout its entire outer surface to the abdominal wall; it was freed by stripping it away, pulled well forwards, and the finger introduced to its own base inside; a ligature was tied round at this point as the finger was withdrawn, and the upper portion entirely cut away. Drainage-tubes were inserted, the edges of the wound brought together with waxed silk sutures, iodoform dusted over, and gauze dressing and a binder applied. The operation lasted one hour and a quarter. Temperature at 12 (midnight) $97^{\circ}$; pulse 84 .

March 4th.-9 A.M.: Pulse 90; temperature 96. The patient has not been sick ; expresses himself as feeling quite comfortable. Ifas had a few teaspoonfuls of wine, water, and milk. Fifteen ounces of urine were drawn off; sp. gr. 1025, acid, no albumen.-3 P.M.: Bowels acted, a copious semi-solid motion being passed.-8 P.M.: Bowels acted again.

5th.-12.30 A.M. : Pulse 96 ; temperature $99^{\circ}$ in mouth. He complains of great thirst; liquids increased, and to take a little barley-water as well.-8.30 P.M.: Temperature $98^{\circ} 8^{\circ}$ Slept several hours. Pulse 100. He has some bronchial catarrh, probably due to ether. Ordered steam inhalations.

6th.-Wound dressed. Total absence of inflammatory swelling; slight serous, inodorous discharge; no tenderness. Evening temperature $100^{\circ}$.

15th.-The patient since last note has steadily improved; temperature normal; pulse 84 ; bowels acted well, and wound granulating freely. Drainage-tubes have been considerably shortened, and the patient is sitting up in bed, going on remarkably well.

\section{CITY OF LONDON INFIRMARY.}

CARCINOMA OF THE LIVER WITH GREAT CONTRACTION OF THE STOMACH, PRODUCING NO SYMPTOMS; NECROPSX; REMARKS.

(Under the care of Mr. W. GEMr.)

GEORGE K-, aged fifty-six, a porter, was admitted into the infirmary on October 29th, 1884, suffering from fistula in ano. His condition on admission was pale and anæmic, with great debility and weakness, although no emaciation was apparent; there was a rather copious discharge from the fistula, which was situated an inch and a half up the bowel. He had faint dulness on deep percussion over the left apex of the lung, but no complaint as regards the abdomen, which was enlarged. The liver dulness somewhat exceeded the normal, and was slightly irregular on the surface, but with no pain or uneasiness. He had a little diarrhoea, which was soon stopped by an astringent mixture. He was placed on a nutritious diet and got slightly better of the discharge and debility, but still complained of lassitude. Operation on the fistula was deferred until his condition became more favourable. Not having improved after being under treatment for a month, he was again thoroughly examined, and on percussion over the iiver complained of pain over the right hypochondrium, with irregular masses slightly increased. All through there was no vomiting or jaundice. The bowels again became free, but were stopped by lead and opium pills. His history, by the way, indicated no hereditary cancer; he was of temperate and regular habits, and had always done his work as a porter without any feeling of illness except the fistula, which troubled him at times. About a month before his death he complained of pain over the right hypochondriac region, increasing slightly, but not to any extent, and a sense of weight and fulness over the epigastrum, but still no vomiting or jaundice occurred. It was only a few days before death that he showed the unmistakable cachexia of cancer, and emaciation became very great, though he still took his food up to the last, without any trouble or vomiting. He gradually got worse, and sank on January 14th last.

The autopsy was made twenty-four hours after death, and revealed the following. The liver appeared double the size of normal in outline, weighed nine pounds, was nodulated on the surface, with flat tuberous masses of cancer of the encephaloid type, and was soft in consistence. The gallbladder was normal in appearance, and contained four drachms of bile. There was, of course, great displacement of the neighbouring viscera. The stomach was contracted so as to be a mere tube of half an inch in diameter. The kidneys 